\title{
Haroldo Dilla
}

\section{Genossen und Investoren Der ungewisse Übergang Kubas}

Vorbemerkung der Redaktion: Der folgende Artikel ist ursprünglich im Socialist Register 1999, S.227-247 veröffentlicht worden. In Kuba selbst ist er nicht erschienen - und er wird dort wohl auch in absehbarer Zeit nicht erscheinen. Stattdessen ist sein Autor, Haroldo Dilla, über ihn ,zum Fall“ geworden: ihm wurde die Entlassung aus seinem Institut angekündigt und er wurde Gegenstand eines Parteiausschlußverfahrens. Dabei ist Dilla ohne Frage einer der renommiertesten Sozialwissenschaftler der Insel, überzeugter Revolutionär und gestandener Marxist. Aber einer, der zwar mit 13 Jahren in Kubas Kommunistischen Jugendverband (UJC) eintrat, aber so viel eigenen Kopf, anti-autoritären Instinkt oder fehlenden Opportunismus zeigte, daß er diesen Schritt gleich noch zwei weitere Male tun mußte, nachdem er 1967 und erneut 1970 wegen „Undisziplin“ beziehungsweise „mangelnden Vertrauens in Fidel und die Partei“ ausgeschlossen wurde. Seit Anfang der 80er Jahre arbeitete Haroldo Dilla am Zentrum für Amerika-Studien (CEA) in Havanna. Als in Kubas Sozialwissenschaften in den 90er Jahren eine lebendige Reformdebatte entstand, war Dilla einer ihrer zentralen Wortführer und intellektuellen Köpfe. In PROKLA 107 ist diese kritische Diskussion, die eine Erneuerung des kubanischen Sozialismus von innen auf die Tagesordnung setzte, dargestellt - und auch die Reaktion der Staatsführung: Armee-Chef Raúl-Castro verlas einen „Bericht des Politbüros“, der in martialischem Ton die Reformdebatte zu Feindeszeug erklärte; im Zentrum für Amerika-Studien (CEA) wurde interveniert: alle seine prominenten Wissenschaftler wurden entlassen und - jeweils einzeln - an andere Einrichtungen versetzt. Haroldo Dilla kam an das Philosophische Institut, das dem Wissenschaftsministerium untersteht. Nach einer solchen Abstrafung gibt es viele Wege, sich wieder zu rehabilitieren oder zumindest unauffällig zu bleiben. Dilla hingegen beharrte darauf, daß die Verhältnisse in Kuba eine kritische, linke Analyse nur um so nötiger machten. Eines der Ergebnisse davon ist der nachfolgende Aufsatz. Dieser Aufsatz war einer der angeführten Gründe für die jüngsten Maßnahmen gegen Dilla. Die Folgen für den Autor sind zur Zeit noch nicht klar absehbar. Sein Parteiausschlußverfahren ist noch in der Schwebe, das letzte Wort der höheren Ebenen steht noch aus. Gegen seine Entlassung aus dem Institut hat Dilla arbeitsrechtliche Schritte eingelegt, die seinen Verbleib zunächst tatsächlich ermöglicht haben. Inzwischen hat er jedoch selbst gekündigt.

Unter dem Applaus von Technokraten und Unternehmern treibt die kubanische Regierung ihre Wirtschaftsreformen voran, wobei sie nicht gerade viele Optionen zur Auswahl hatte. Die Desintegration des osteuropäischen Wirtschaftsblocks und danach der UdSSR markierte einen schweren Schlag für die Wirtschaft Kubas. Stagnierte die Wirtschaft der Insel schon zwischen 1986-1989, so begann ab 1990 eine rasante Talfahrt, die bis $1993 \mathrm{zu}$ einer Abnahme des Bruttoinlandsproduktes um nahezu 40\% führte. Beim Außenhandel ergibt sich ein noch dramatischeres Bild, da das Land unver- 
mittelt $85 \%$ seiner traditionellen Märkte, nahezu seine gesamten kurz- und mittelfristigen Kredite sowie seine wichtigsten Quellen technologischen Nachschubs verlor; die Importkapazität der Wirtschaft reduzierte sich um zwei Drittel.

In einer solchen Situation begann die sonst dem Markt eher mißtrauisch gegenüberstehende kubanische Regierung mit der pragmatischen Duldung diverser Realitäten: Kuba mußte sich unter rigiden Wettbewerbsbedingungen in den kapitalistischen Weltmarkt einfügen und dafür die Fundamente seiner ökonomischen und sozialen Organisation ändern. Am 26. Juli 1993 bekannte sich der kubanische Präsident in einer denkwürdigen Rede zu den Einschränkungen, die ein solches Programm mit sich bringt: es gehe darum, die revolutionären Errungenschaften zu wahren und bessere Zeiten abzuwarten, um dann den sozialistischen Aufbau fortzusetzen.

Trotz bürokratischer Hindernisse und nicht wenigen Rückschritten zeigte der wirtschaftliche Anpassungs- und Liberalisierungsprozeß eine überraschende Dynamik. Schon 1987 wurde ein Förderprogramm für ausländische Investitionen eingeleitet. Diese genießen seit 1995 durch ein eigens geschaffenes Gesetz einen ausgedehnten Schutz. 1992 wurde eine substantielle Verfassungsreform verabschiedet, die das Privateigentum an Produktionsmitteln anerkennt und durch die Abschaffung des staatlichen Außenhandelsmonopols Möglichkeiten zur Dezentralisierung der Staatsunternehmen eröffnet. Ein Jahr später, während des rapiden Absturzes des Bruttoinlandsproduktes, wurde der gesamten kubanischen Bevölkerung der Besitz von Dollars und anderen harten Währungen erlaubt, mit denen auch in Läden eingekauft werden konnte, die bisher nur den im Land lebenden Ausländern, Touristen und einem begrenzten Teil der nationalen Elite vorbehalten waren. Zum selben Zeitpunkt wurde ein beträchtlicher Teil des staatlichen Landbesitzes in die Verantwortung von Kooperativen und in geringerem Umfang auch in die von Kleinbauern überführt. Zusätzlich wurde die Selbständigkeit im Dienstleistungsbereich mittels einer vielversprechenden Gesetzgebung gefördert.

Mit dem Ziel, einen mittlerweile horrenden Geldüberhang abzubauen, begann 1994 eine Politik der Haushaltssanierung, der Preiserhöhungen und der Einführung von Steuern und anderen Abgaben. In der zweiten Hälfte desselben Jahres legalisierte der Staat die freien Bauern- und Lebensmittelmärkte, deren Abschaffung im Jahr 1986 Ausgangspunkt der letzten politischen Kurskorrektur in Kuba gewesen war. Da die staatlich subventionierte Lebensmittelversorgung beständig eingeschränkt worden war, deckte dieser freie, vom Dollar dominierte Markt schon damals einen beachtlichen Teil des Konsums der Bevölkerung, wobei sich die Preise aber nicht an den Arbeitslöhnen orientierten. Zwischen 1996 und 1997 wurden die vorangegangen Reformschritte konsolidiert: in vier Regionen wurden Freihandels- 
zonen eingerichtet, die Zollgesetze wurden modifiziert und das Bankwesen wurde reorganisiert.

Die politische Führung Kubas proklamierte während dieser schweren Zeit wiederholt die Erfolge ihrer Öffnungspolitik: in erster Linie gelang es ihr, den katastrophalen Wirtschaftseinbruch aufzuhalten und seit 1995 erhebliche Wachstumsraten zu erreichen. Darüber hinaus wurde die Hoffnung der amerikanischen Ultrarechten und ihrer Verbündeten in Miami jäh zerschlagen, daß eine wirtschaftlich kollabierte Revolution schließlich zur Kapitulation gezwungen wäre. Berücksichtigt man, daß diese Ergebnisse inmitten einer harten Konfrontation mit den USA erreicht wurden, deren Wirtschaftsblockade ein Katalysator der Krise war, dann muß man den Reformanstrengungen eine historische Bedeutung beimessen.

Nach offizieller Lesart wurde die wirtschaftliche Erholung erreicht, ohne sich der neoliberalen Orthodoxie zu verschreiben: der Staat behielt seine wichtige Rolle, Gesundheits-, Bildungs- und Sozialprogramme wurden nicht geopfert, ihre Budgets blieben stabil oder wuchsen sogar an (sie machen insgesamt circa 60\% des Staatshaushaltes aus). Auch hier handelt es sich um ein Verdienst, das anerkannt werden muß und den sozialen Charakter der Revolution unter Beweis stellt.

Aber auch für Beobachter, die sich zur politischen Linken zählen und die von der historischen Bedeutung der kubanischen Revolution überzeugt sind, bleiben zentrale Fragen unbeantwortet. Der ökonomische Liberalisierungsprozeß in Kuba unterscheidet sich von den laufenden Entwicklungen in Lateinamerika nicht nur aufgrund der oben genannten Aspekte, sondern auch dadurch, daß es sich im Falle Kubas nicht um eine Anpassung bereits existierender kapitalistischer Verhältnisse handelt. Es geht vielmehr um eine radikale Restrukturierung der politischen Ökonomie, der Formen sozialer Regulierung und der ideologisch-kulturellen Produktion, die in den vergangenen Jahrzehnten prägend waren. Dies impliziert aber eine qualitative Umgestaltung: die zunehmende Kommerzialisierung der sozialen Aktionsfelder wirft auf verschiedenen Ebenen die zentrale Frage der Politik auf die Frage nach der Verteilung der Macht. Wenn wir einmal unterstellen, $\mathrm{da} ß$ die Kombination von militantem Antiimperialismus und kostenlosen Sozialsystemen nicht schon Sozialismus ist, dann stellt sich die Frage nach der Reichweite der Systemveränderungen - und zwar sowohl auf der gesellschaftlicher Ebene (hinsichtlich der Klassenstruktur) wie auch hinsichtlich der gesamten Reartikulation des politischen Lebens.

Im folgenden soll gezeigt werden, daß die aktuellen Reformen die Klassenverhältnisse zugunsten eines technokratisch-unternehmerischen Blockes und zum Nachteil der Bevölkerungsmehrheit verschieben. Diese Entwickung hin zu einer kapitalistischen Restauration (die im Namen des Sozialismus und unter Führung der Kommunistischen Partei erfolgt) ist aber nicht unausweich- 
lich. Es existieren alternative Wege, die am Sozialismus festhalten, die aber von einem alternativen linken Projekt auf internationaler Ebene nicht zu trennen sind, und die in Kuba eine substantielle Erneuerung des politischen Systems zugunsten einer genuinen Volksmacht implizieren würden.

\section{Das Szenario von einst: die subventionierte Utopie}

Die soziale Dynamik Kubas zwischen 1959-1989 war von zwei gegensätzlichen Tendenzen geprägt: einerseits von gesellschaftlicher Nivellierung und andererseits von sozialer Mobilität. Erstere dominierte während der frühen Revolutionsjahre und hatte ihren Ausgangspunkt im Radikalismus der Revolution, die zum Verschwinden der Bourgeoisie und weiter Teile der Mittelschicht führte, welche emigrierten oder einen Prozeß der Proletarisierung erfuhren. Die Gesellschaft organisierte sich allmählich entlang der nun dominierenden gesellschaftlichen und staatlichen Eigentumsformen. Zugleich wurde die gesellschaftliche Nivellierung durch eine zunehmende soziale Mobilität (besonders seit Mitte der 70er Jahre) begleitet, die sich auf staatliche Programme zur Schaffung von Arbeitsplätzen und dem Ausbau von sozialen Dienstleistungen stützte. So wohnten z.B. 1953 53\% der Bevölkerung in urbanen Gebieten, die Analphabetenquote betrug etwa 25\% und nur $11 \%$ der Bevölkerung hatte die Mittlere Reife oder die Hochschulreife. 1989 hingegen, dem letzten Jahr, in dem ein statistisches Jahrbuch in Kuba veröffentlicht wurde, zählte man etwas mehr als 10,5 Millionen Einwohner, von denen $73 \%$ in urbanen Gebieten lebten. Ungefähr 38\% der Gesamtbevölkerung wohnte in Städten mit mehr als 100.000 Einwohnern. Analphabetismus gehörte der Vergangenheit an. Mehr als die Hälfte der Bevölkerung besaß eine Schulbildung von mehr als sechs Schuljahren, die Anzahl an Technikern und Akademikern erreichte eine halbe Million und ungefähr 140.000 Menschen waren in höheren Bildungsinstituten eingeschrieben (Anuario de 1989; Comunicado 1983).

Diese intensive soziale Mobilität führte zwar zu einer Differenzierung der Subjekte, die einzelnen gesellschaftlichen Bereiche entwickelten aber keine eigene soziale Identität. Die soziale Identität war vor allem vom Konzept „Volk“ (el pueblo) als dem Subjekt der sozialen Transformation und der nationalen Verteidigung bestimmt. Das politische System reflektierte dies durch seine Anlehnung an das leninistische Modell der sektoral strukturierten Massenorganisationen, welche als „Transmissionsriemen“ zwischen der Gesamtheit der Bevölkerung und der innerhalb der kommunistischen Partei organisierten „Avantgarde“ fungieren sollten. Das Ergebnis war eine starke Konzentration der politischen Autorität. Die revolutionäre politische Klasse verfügte zumindest in drei Richtungen über ein Monopol bei der Regulierung des gesellschaftlichen Lebens. 
Erstens war die Macht, die ökonomischen Ressourcen durch einen einzigen, zentralisierten Planungsmechanismus zu verteilen, durch den Eintritt Kubas in den sowjetischen Block Anfang der 70er Jahre noch weiter gestärkt worden. Von jenem Zeitpunkt an bis zum Jahr 1987 war die kubanische Wirtschaft durch extensives Wachstum bei hohem Ressourcenverbrauch und einer nur wenig effizienten Produktion, bei annähernd gleichen Verteilungsverhältnissen und subventioniertem Konsum gekennzeichnet. Zweites wurde das leninistische Modell der politischen Organisation - getragen von einem starken politischen Konsens - zu einem wichtigen Mechanismus politischer Kontrolle, und zwar nicht nur in bezug auf die Unterdrückung oppositioneller Tendenzen, die es seit Anfang der 60er Jahre kaum noch gab, sondern insbesondere zur Mobilisierung der Massen und zur Verbreitung von politischen Werten und Einstellungen.

Ein dritter, nicht minder wichtiger Punkt, war die Fähigkeit der politischen Führung, eine glaubhafte Legitimationsideologie zu schaffen. Es handelte sich um eine in sich geschlossene Ideologie, die wenig Raum für Ungewißheiten bezüglich der dreifachen Frage nach dem Existierenden, dem Besten und dem Möglichen ließ. Sie war kohärent, wenn man berücksichtigt, daß zwischen der Wahrnehmung der alltäglichen Realitäten, wie sie sich innerhalb der sozialen Strukturen zeigte, und den institutionalisierten Diskursen eine enge Wechselbeziehung bestand; diese Ideologie konnte auch der durchschnittliche Bürger akzeptieren, der Werte und Prinzipien wie Patriotismus, Internationalismus, soziale Gleichheit etc. teilte, die von der nationalen politischen Kultur hochgehalten wurden. Der Kapitalismus wurde mit all seinen organischen Bestandteilen wie Bourgeoisie, Konsumismus, Ungleichheit, Markt etc. verdammt und als Teil einer Vergangenheit betrachtet, die keine zweite Chance bekommen würde.

Dieses Schema beinhaltete jedoch ernsthafte Widersprüche, die aus dem erklärten Ziel einer „Vergesellschaftung“ der Macht, und ihrer tatsächlichen Aneignung durch eine bürokratische Schicht resultierten. Letztere entstand während der 60er Jahre und konsolidierte sich endgültig im folgenden Jahrzehnt. Die Etablierung dieser Schicht war nur möglich mittels einer Ausdehnung klientelistisch-paternalistischer Beziehungen, wodurch die Vergesellschaftung der Macht verzögert wurde, was zur Folge hatte, daß die sozialistischen Entwicklung stagnierte (vgl. dazu Dilla 1996).

Die Geschichte hat sowohl die Vor- als auch die Nachteile einer solchen Ordnung demonstriert. Solange es sich um eine Gesellschaft handelte, die ein niedriges Niveau von „Universalisierung und sozialer Reflexität“ aufwies (um die Ausdrücke von Giddens 1997 zu benutzen), funktionierte dieses Schema der politischen Regulation effizient: es erlaubte, sich der externen Gefahr, die von den USA ausging, mit Erfolg entgegenzustellen, es erleichterte die Mobilisierung und die egalitäre Verteilung der verfügbaren 
Ressourcen und es förderte unter anderem eine solidarische politische Kultur sowie ein weitgespanntes Netz sozialer Partizipation und Mobilisierung. Aber die eigenen Erfolge führten auch zum Erschlaffen des Modells, als nämlich die Mobilität und die hohe Bildung der Menschen mit den rigiden Mechanismen der politischen Kontrolle zusammenstieß, was zu Dysfunktionen wie Gleichgültigkeit und Politikverdrossenheit führte. Die wirtschaftlichen Reformen besorgten dann den Rest. Der Markt, der in früheren Jahrzehnten von eher untergeordneter Bedeutung war, begann eine stärkere Rolle bei der Verteilung der Ressourcen und des mageren wirtschaftlichen Überschusses zu spielen; und er tat dies zwangsläufig auch bei der Neuordnung der Machtverhältnisse. Die Durchschnittskubaner entdeckten mit Bestürzung, daß die Zukunft nicht so sicher war, wie über viele Jahre hinweg in öffentlichen Debatten behauptet worden war.

\section{Gesellschaft, Politik und Machtverhältnisse in der neuen Ära}

Eine der Konstanten des offiziellen kubanischen Diskurses bestand in der Überzeugung, daß die politischen Strukturen in regelmäßigen Abständen $\mathrm{zu}$ erneuern sind. Allerdings war diese Erneuerung in der Praxis durch zahlreiche Faktoren begrenzt, angefangen von bestimmten Vorgaben - wie beispielsweise dem Einparteiensystem - bis hin zur direkten Ablehnung jeglicher von außen induzierter Veränderungen. Dies erscheint angesichts der nordamerikanischen Bestrebungen, das politische System Kubas so umzugestalten, daß die USA wieder eine entscheidende Rolle in den heimischen Angelegenheiten erlangen könnten, sehr verständlich.

Das Jahr 1990 kennzeichnete ein bedeutendes Moment innerhalb der Dynamik der politischen Erneuerung. Es war nicht unbedingt ein ,gutes Jahr“. Die Wirtschaft litt seit 1987 unter einem stetigen Verfall, der auch durch den von der Revolutionsführung proklamierten Kurswechsel (der ,rectificación") nicht aufgehoben werden konnte. Das ideologische Konstrukt der Unumkehrbarkeit der sozialistischen Entwicklung erlitt einen schweren Rückschlag: zunächst durch die Auswirkungen der Perestroika, dann durch den Fall der Berliner Mauer. In der zweiten Hälfte von 1989 wurde die kubanische Gesellschaft außerdem durch Korruptionsvorwürfe gegenüber hohen Amtsträgern innerhalb des Militärs, der Staatssicherheit sowie des zivilen Sektor erschüttert.

Die Notwendigkeit, wieder einen Konsens zu erlangen und damit die Legitimität des Regimes zu stärken, wurde im Vorfeld des 1991 geplanten IV. Kongresses der Kommunistischen Partei von der politischen Klasse als eine nicht aufzuschiebende Aufgabe angesehen. Als Konsequenz rief die Partei zu einer öffentliche Debatte auf, die

„,...einen Konsens fördern sollte, der auf der Anerkennung der Meinungsvielfalt, die im Volk existiert, basiert und der durch eine demokratische Diskussion im Innern der Partei und der 
Revolution bestärkt wird, vor allem bei der Suche nach Lösungen, der Überprüfung von Alternativen, die sich zur Erreichung von sozioökonomischen Zielen anbieten und im allgemeinen hinsichtlich der Überlegungen, die darauf ausgerichtet sind, die Gesellschaft, in der wir leben, zu verbessern“ (Llamamiento 1990).

Im Verlauf mehrerer Monate erlebte die kubanische Gesellschaft die freieste und demokratischste öffentliche Debatte ihrer Geschichte. Millionen von Menschen übten an vielen tausend Orten (Schulen, Arbeitsstätten, Gemeinden) ihr Recht aus, Kritik zu üben, Lösungsvorschläge zu unterbreiten oder schlicht über Themen zu beraten, die vom täglichen Dasein bis hin zum hohen politischen Tagesgeschäft reichten. Die Ergebnisse dieser Auseinandersetzung wurden nie veröffentlicht, aber einzelne Berichte und Beobachtungen legen den Schluß nahe, daß die sozialistischen Ziele und die nationale Unabhängigkeit nicht in Frage gestellt wurden, daß aber in diesem Rahmen eine grundlegende Systemerneuerung gefordert wurde. Die politische Klasse Kubas hatte eine Flut an Informationen vor sich, die ausreichend waren, um den Gemütszustand, die Hoffnungen und die Sichtweise eines Großteils der Bevölkerung einzuschätzen.

Die politischen Veränderungen der nächsten zwei Jahre (1991-1992) versuchten diesen Forderungen nachzukommen, aber die eingeleiteten Maßnahmen waren nur noch ein schwaches Echo der intensiven Debatten. Zunächst wurde eine substantielle Reform der Verfassung durchgeführt, die circa $60 \%$ des Artikelwerkes modifizierte, und obwohl sich ein großer Teil der Änderungen auf die ökonomische Sphäre bezogen (Eigentumsformen, Entflechtung des Außenhandels, usw.), betrafen andere Modifizierungen (zumindest als Absichtserklärungen) das, was Azcuy (1996) „den harten Kern“ der Verfassung von 1976 nennt: die Proklamation des nicht-konfessionellen Charakters des Staates und das Verbot jeglicher Diskriminierung gegenüber Gläubigen; Verzicht auf die Erwähnung des demokratischen Zentralismus und der Einheit der Gewalten; Aufhebung der klassenspezifischen Definition der sozialen Basis des Staates; Etablierung von Direktwahlen für parlamentarische Sitze. Ein neues Wahlgesetz trug vor allem dem letzten Vorschlag Rechnung. Doch obwohl bei der Nominierung der Kandidaten die direkte Einmischung der Kommunistischen Partei gebremst und die Rolle der sozialen Massenorganisationen gestärkt wurde, war das neue Verfahren durch die Einschränkung des Wettbewerbsprinzips dennoch in seiner demokratischen Reichweite begrenzt. Gleichzeitig wurden neue Gemeinde-Unterorganisationen - sogenannte Volksräte - geschaffen, die eine wichtige Rolle bei lokalen Fragen spielten. Was einige bereits als Anzeichen des Aufbaus einer pluralistischen und partizipatorischen Demokratie in sozialistischer Richtung angesehen haben, wurde jedoch durch eine Reihe von Veränderungen begrenzt, die mehr an einer effektiven Regierungsarbeit als an der Demokratie orientiert waren. 
Wenn Politik nicht ausschließlich institutionell oder normativ bestimmt ist, sondern - um mit Held (1992) zu sprechen - es hauptsächlich mit einer Interaktion verschiedener Akteure zu tun hat, bei der es um die Kontrolle der Allokation von Ressourcen und Werten geht, dann muß zugegeben werden, daß in Kuba die Politik gegenüber den Anforderungen des neuen Akkumulationsmodells allmählich zu erodieren beginnt, was seinen deutlichsten Ausdruck in der Veränderung der gesellschaftlichen Machtstrukturen findet. Daher sind die formalen Veränderungen, die heutzutage noch vorgenommen werden können, ganz anders als die, die in Zukunft noch möglich sein werden, wenn sich die Machtverhältnisse signifikant verändert haben werden. Es zeigt sich nämlich eine doppelte Tendenz: einerseits die Stärkung eines an den Markt gekoppelten technokratisch-unternehmerischen Blocks, der sich in einen hegemonialen Block verwandeln kann, andererseits eine Fragmentierung und Schwächung der breiteren Gesellschaftsschichten.

\section{Das Recycling der Eliten}

Das herausragendste soziale Ergebnis des ökonomischen Öffnungs- und Reformprozesses ist die beginnende Formierung eines neuen sozialen Blocks, den ich als technokratisch-unternehmerisch bezeichne und der aus drei Komponenten besteht.

Die erste Komponente findet sich im Umkreis der Auslandsinvestitionen. Nach den zur Verfügung stehenden Zahlen gab es 1990 nicht einmal zwei Dutzend ausländische Investoren in Kuba. Im Jahre 1994 existierten 174 Unternehmen mit ausländischem Kapital, deren Investitionen sich auf 1,5 Mrd. US-Dollar beliefen. Sie stammten aus 36 Ländern und waren in 26 Branchen tätig. Gleichzeitig zählte man die Anwesenheit von 400 Handelsvertretungen. ${ }^{2}$ Bis Ende 1996 war die Zahl der Auslandsinvestoren auf 260 gestiegen, einige von ihnen begannen sich in den neuen Freihandelszonen niederzulassen. In jenem Jahr wurde die Anwesenheit von ungefähr 800 ausländischen Firmenvertretungen gezählt (Granma, 14. 12. 1996). In der

1 An dieser Stelle ist eine Entschuldigung angebracht: in Kuba werden seit 1989 keine offiziellen Statistiken mehr publiziert, so daß sich die folgenden Analysen auf Teilstudien stützen müssen, die natürlich immer Platz für Zweifel lassen.

2 Diese Daten wurden auf der XII. Internationalen Messe in Havanna veröffentlicht. Bei der Eröffnungsfeier versicherte eine hohe politische Führungsperson den dort Versammelten: „Wir bieten ihnen ein geordnetes Land, eine kohärente und unumkehrbare Politik der Öffnung gegenüber Investitionskapital, eine zusammenhängende und ausgebaute ökonomische Infrastruktur, einen effizienter werdenden Produktionssektor, ein fleißiges und selbstloses Volk mit einem hohen Stand von Bildung und Technik und eine Gesellschaft, die keinen Terrorismus und keine Drogen kennt. Wir bieten Ihnen eine souveräne Nation und eine ehrenhafte und nicht korrupte Regierung." (vgl. Cuba Foreign Trade, Juli-Dezember 1994). 
Regel handelt es dabei um Gemeinschaftsunternehmen mit dem Staat, so daß dieser Sektor eng mit einer Schicht von Unternehmern und nationalen Führungskräften verbunden ist, die Lebensweisen und Bestrebungen teilen, die sich substantiell von dem Rest der Bevölkerung unterscheiden.

Die zweite Komponente dieses aufstrebenden Blocks besteht aus den Führungskräften von staatlichen Unternehmen, die vorteilhafte Positionen auf dem Weltmarkt erzielt haben und die infolgedessen eine größere Autonomie besitzen. Deren neue Vollmachten sind unvereinbar mit der traditionellen Figur eines Unternehmensleiters des öffentlichen Sektors in einer zentralen Planwirtschaft, der immer in der tragischen Dreifaltigkeit des NichtWissens, Nicht-Könnens oder Nicht-Wollens eingeschlossen ist. Statt dessen wächst ein neuer Typ von nationalen Unternehmern heran, die sich mehr um Gewinnmaximierung kümmern als um politische Erwägungen. Mit der Weiterführung der Wirtschaftsreformen wird auch die Zahl solcher Unternehmen ansteigen.

Die dritte Komponente dieses Blocks wird durch diejenigen Personen repräsentiert, die hohe Geldsummen und Güter durch Schwarzmarktspekulationen angehäuft haben, oftmals auf Kosten der staatlichen Ressourcen (wohlhabende Bauern, kommerzielle Zwischenhändler, Dienstleistungsanbieter etc.). Da der Großteil dieser Einnahmen einen illegalen Ursprung hat, ist es unmöglich, ihr ökonomisches Potential genau zu bestimmen. Aber es ist möglich, die Entwicklung durch eine Analyse der Geldkonten, die circa $60 \%$ der gesamten Geldliquidität binden und die in den letzten Jahren beunruhigende Konzentrationstendenzen zeigten, annähernd $\mathrm{zu}$ erfassen. Nach den von der kubanischen Nationalbank und anderen offiziellen Agenturen veröffentlichten Berichten verfügten Ende 1994 14,1\% der Bankkonten über 77,8\% aller Ersparnisse. Ein Jahr später befanden sich auf 13,1\% der Konten 83,7\% der Ersparnisse und 1996 war diese Konzentration so weit angestiegen, daß auf 12,8\% (etwas mehr als 600.000 Konten) $84,7 \%$ der Gesamtersparnisse verteilt waren, mehr als 6,6 Milliarden kubanische Pesos. Noch signifikanter ist vielleicht, daß sich 1996 auf nur 2,7\% der Konten 43,8\% der Ersparnisse befanden. In jenem Jahr sanken die Einlagen der Konten mit Einlagen bis 20.000 Pesos um fast 300 Millionen Pesos, während die Konten über 20.000 Pesos ihren Wert um 74,1 Millionen Pesos erhöhten.

Mit der Liberalisierung der Agrar- und Industriemärkte ebenso wie mit der Zulassung von Selbständigen wurde dieser Sektor nicht nur gestärkt und konnte seine Einkommen verbessern und legalisieren, er erlangte auch eine größere Kontrolle über die Bewegungen auf dem Binnenmarkt. In naher Zukunft wird dieser Sektor zu einem wichtigen Investor für kleinere und

3 Konten in harter Währung gab es nur 4.500 mit ca. 9,5 Millionen Dollar (Beruff 1997). 
mittlere Betriebe werden sowie vertragliche Verbindungen mit dem formalen Bereich der Wirtschaft aufnehmen, was wiederum seine Akkumulationsmöglichkeiten erweitern wird.

Wie man sich leicht vorstellen kann, entstammen die sozialen Gruppen dieses neuen Blocks, insbesondere die ersten beiden, der traditionellen - zivilen oder militärischen - Bürokratie der Insel, bzw. aus deren Familien. Selbst innerhalb der dritten Gruppe ist es möglich, eine starke Verbindung zwischen den lukrativsten Privatgeschäften - Restaurants und Pensionen für Touristen - und hochrangigen pensionierten Bürokraten oder deren Familienangehörigen $\mathrm{zu}$ finden, denn solche Geschäfte benötigen komfortable und zentral gelegene Wohnungen, die vom Staat früher oft an solche Personen vergeben wurden.

\section{Die gesellschaftliche Fragmentierung}

Vor 1989 war die kubanische Arbeiterklasse eine relativ homogene Masse. In jenem Jahr waren ca. 3,5 Millionen Personen, d.h. 94\% der gesamten im zivilen Sektor beschäftigten Arbeitskräfte, staatliche Lohnarbeiter, die zum allergrößten Teil gewerkschaftlich organisiert und durch ein paternalistisches Arbeitsschutzgesetz geschützt waren. Die Zahl der Selbständigen belief sich auf wenige tausend, die Zahl der Privat- oder Genossenschaftsbauern war gering und am Schrumpfen. Die Krise und der Liberalisierungsprozeß haben diesen Zustand wesentlich verändert.

Die lohnabhängigen Schichten haben sich in Folge der Entstehung neuer und lukrativer Arbeitsmöglichkeiten im privaten oder kooperativen Sektor und aufgrund des Arbeitsplatzabbaus in den Staatsbetrieben absolut verringert. Im Jahre 1996 zählte der staatliche Sektor noch 78\% der ökonomisch aktiven Bevölkerung, 16\% weniger als acht Jahre zuvor.

Nicht weniger schwerwiegend war die wirtschaftliche Schwächung dieses Sektors: einerseits durch die Dollarisierung der Preise eines bedeutenden Teils der Konsumgüter und der Dienstleistungen und andererseits durch die weiterbestehende Koppelung der Gehälter an das Preisniveau des subventionierten Konsums. In diesem Sinne unterliegen die staatlichen Arbeitsverhältnisse einer übermäßigen Ausbeutung, da der Preis der Arbeitskraft geringer ist als ihre Reproduktionskosten. Nach inoffiziellen Kalkulationen (Togoros 1997) benötigt eine vierköpfige kubanische Familie, in der zwei Personen arbeiten und ein durchschnittliches Einkommen erhalten, das Doppelte dieses Einkommens für den Mindestkonsum an Nahrungsmitteln, Hygieneartikeln und Dienstleistungen.

Allerdings sind auch diese Angaben noch recht ungenau. Fast 20\% der Lohnabhängigen, die in den jetzt privilegierten Bereichen (Tourismus, Hochtechnologie, Exportindustrien) arbeiten, erhalten neben dem offiziel- 
len Gehalt noch weitere Einkünfte, was zu einer schleichenden Umstrukturierung der Arbeiterklasse durch das internationale Kapital führt.

In anderen Fällen haben sich die Arbeiter selbständig gemacht, sei es durch formale Antragstellung (fast $26 \%$ der ausgestellten Gewerbelizenzen haben staatliche Arbeiter erhalten) oder durch informelle Tätigkeiten. Allerdings gibt es häufiger auch andere, nicht an Erwerbsarbeit gebundene Überlebensstrategien, wie etwa Korruption oder Zuwendungen von emigrierten Familien.

Ebenfalls wichtig ist die Vermehrung der privaten Kleinbauern und der genossenschaftlich organisierten Agrarproduzenten. Diese Sektoren hatten sich seit dem Triumph der Revolution kontinuierlich verringert: 1970 umfaßten sie noch $11 \%$ der Beschäftigten des zivilen Sektors, 1989 nur noch 5\%. Mit der Gründung von Agrarkooperativen hat diese Situation eine dramatische Wende erfahren, und obwohl keine offiziellen Statistiken existieren, wird davon ausgegangen, daß sich der landwirtschaftliche Genossenschaftssektor um fast 300.000 Personen vergrößert hat und über 30\% der gesamten Agrarfläche verfügt. Außerdem haben weitere 50.000 Personen private Eigentumstitel erhalten (Valdés 1997).

Ein anderer Sektor, der sich insbesondere in urbanen Zonen beträchtlich ausdehnte, sind die Selbständigen. Selbständiges Arbeiten war in der kubanischen Gesellschaft nicht völlig unbekannt. In den 70er Jahren hatte diese Art von Aktivitäten eine gewisse Verbreitung erreicht, die zwischen 1986 und 1989 wieder eingeschränkt wurde, als nach dem Kurswechsel des sogenannten ,proceso de rectificación“ Selbständigkeit für unvereinbar mit den sozialistischen Ziele angesehen wurde. Im Sommer 1993 wurde die Selbständigkeit als Arbeitsbeschaffungsmaßnahme und um den Schwarzmarkt auszutrocknen rehabilitiert. Obwohl sich in der erlaubten Form der Selbständigkeit, dem sogenannten „cuentapropismo“ bedeutsame Einkommenschancen verbergen, sind die meisten der Geschäfte, die unter dieses Gesetz fallen, kleine individuelle oder familiäre Einheiten, dessen Nettoeinkünfte, auch wenn sie die Löhne der meisten Arbeiter des formalen Sektors übersteigen, keine Basis zur Akkumulation bieten.

Im Februar 1994 existierten 142.000 registrierte Selbständige, eine Zahl, die bis zum Juni auf 160.000 und im Januar 1996 auf 208.346 gestiegen war. 1997 verzeichnete man ein signifikantes Schrumpfen auf etwas mehr als 160.000 Personen. Diese Zahlen spiegeln jedoch nicht die wahre Dynamik des Prozesses wider. Bis 1997 wurden insgesamt 401.847 Genehmigungsanträge gestellt, und gleichzeitig gaben 158.597 Personen ihre Selbständigkeit auf, was auf eine hohe Instabilität verweist. Nur $0,9 \%$ aller Selbständigen waren im lukrativsten Bereich, den Privatrestaurants tätig, $27 \%$ widmete sich an Ständen dem Verkauf von Lebensmitteln und nichtalkoholischen Getränken. Zum genannten Zeitpunkt waren 26\% der Selb- 
ständigen gleichzeitig Arbeiter im formalen Sektor, 30\% waren arbeitslos, 18\% Hausfrauen (ein oft benutzter Euphemismus für arbeitslose Frauen) und der restliche Anteil bestand aus Rentnern. 73\% der Lizenzinhaber waren Männer; besonders interessant ist, daß $80 \%$ der registrierten Selbständigen mehr als neun Jahre Schulbildung hatte.

Abschließend muß erwähnt werden, daß sich die kubanische Gesellschaft von einem Zustand der Vollbeschäftigung (die ursprünglich als eine revolutionäre Errungenschaft betrachtet wurde, selbst wenn sie nur um den Preis ökonomischer Ineffizienz erreichbar war) auf eine Situation hin bewegt, in der Arbeitslosigkeit zu einem strukturellen Problem wird. 1994 lag die offizielle Arbeitslosenquote bei 8,5\%, 1997 bei 7\%, was von einigen Analytikern als positives Zeichen angesehen wurde. Allerdings war dieser Rückgang nur durch eine graduelle Anwendung und teilweise sogar durch eine branchenübergreifende Zurückstellung von betrieblichen Rationalisierungsmaßnahmen möglich. Im Rahmen dieses Gradualismus gab es neue Optionen, insbesondere im wachsenden privaten Sektor. Es wird davon ausgegangen, daß 70\% der im staatlichen Sektor freigesetzten Arbeitskräfte vom Privatsektor absorbiert wurden. Es ist auch möglich, daß die künftige Zulassung kleiner und mittlerer Unternehmen weitere Ventile in diese Richtung öffnet. Aber es handelt sich dabei nicht um unbegrenzte Möglichkeiten, so daß die Arbeitslosenzahl in den nächsten Jahren selbst dann weiter steigen wird, wenn die Wirtschaft ein nennenswertes Wachstum erreicht, denn eine Vorbedingung für die wirtschaftliche Reproduktion des neuen Akkumulationsmodells ist die Entlassung der überzähligen Arbeitskräfte.

\section{Die Zukunft von links denken}

Die Zukunft Kubas aus einer linken Perspektive zu überdenken, ist eine Notwendigkeit, die weit über die nationalen Grenzen hinausgeht. Der Kampf des kubanischen Volkes für seine nationale Unabhängigkeit und seine sozialen Errungenschaften wird auf der gesamten Welt anerkannt und hat eine Solidaritätsbewegung von großer moralischer und politischer Bedeutung hervorgerufen. Genauso beachtenswert ist die Weigerung der kubanischen Führung sich dem Imperialismus zu unterwerfern. All das ist

4 Vgl. dazu die kritische Analyse von García (1996). Die Daten über Selbständige müssen mit Vorsicht genossen werden, da es üblich ist, daß hinter jedem registrierten und steuerzahlenden Selbständigen mehrere mitarbeitende Personen stehen. Deshalb ist die Zahl der derjenigen, deren Haupteinkünfte aus der Selbständigkeit kommen, erheblich höher.

5 Vgl. Togoros (1997) sowie Granma, 26.11. 1997. Die offiziellen Arbeitslosenzahlen sind stets zu klein, da sie sich nur auf Personen beziehen, die sich registrieren ließen, was aber nur ein Teil der Betroffenen tut. Außerdem gibt es keine Zahlen über die Unterbeschäftigung. Von Arbeitslosigkeit sind vor allem Jugendliche unter 30 und Frauen betroffen. 
wichtig, aber noch nicht ausreichend. Denn Kuba bietet noch eine weitere Chance: Kuba könnte zum Bestandteil eines sich entwickelnden antikapitalistischen Projekts werden, das - auch wenn es viele national unterschiedliche Gesichter hat - nur auf einer internationalen Ebene lebensfähig sein kann. Aber um dies zu erreichen, muß Kuba nicht nur seine sozialen Errungenschaften und seine Unabhängigkeit bewahren, es muß auch ein neues Entwicklungs- und Politikkonzept geschaffen werden, eine genuine, demokratische und pluralistische Volksmacht. Die kubanische Gesellschaft hat dafür durchaus die Potentiale: sie besitzt ein starkes Netzwerk an Partizipationsmöglichkeiten, eine politische Kultur, für die Solidarität und Kooperation von zentraler Bedeutung ist, Menschen, die sich an Werten orientieren, die zur Erreichung sozialistischer Ziele notwendig sind sowie eine politische Klasse, die in Teilen mit Sinn für Verantwortung und einer hohen sozialen Sensibilität ausgestattet ist. Es gibt allerdings auch Hindernisse: insbesondere die schwierigen Bedingungen, unter denen das Land eine wirtschaftliche Erholung anstrebt, die asymmetrischen Beziehungen zum kapitalistischen Weltmarkt und schließlich die wirtschaftlichen und politischen Auswirkungen der nordamerikanischen Blockadepolitik.

Da eine rein autarke Entwicklung nicht möglich scheint, ist die Wiedereingliederung Kubas in den kapitalistischen Weltmarkt eine unabdingbare Voraussetzung für das nationale Überleben. Dies impliziert aber keineswegs die fatalistische Anerkennung der Spielregeln der sogenannten Globalisierung, und noch viel weniger, daß es nicht möglich wäre, alternative Wege zu finden. Aber realistischerweise läßt sich nicht leugnen, daß die Integration in den Weltmarkt, wie immer sie auch erfolgen mag, eine dramatische Veränderung in den Machtbeziehungen und der Handlungsweise des Staates mit sich bringen wird. Damit wird aber auch eine Neugestaltung der Politik notwendig, die auf drei grundlegenden Beziehungen beruhen sollte. Erstens muß die Einheit der Nation gegenüber jeglichen imperialistischen Einmischungen garantiert werden. Zweitens müssen das komplexer werdende „Volk“ und seine Organisationen gestärkt werden. Drittens muß auf die sozialen Unterschiede mit einer Hegemonie des „Volkes“ reagiert werden, der sich auch die aufstrebenden Sektoren in einer noch auszuhandelnden Weise unterzuordnen haben. Zusammenfassend würde es sich um ein Paradigma sozialistischer Politik handeln, welches die Existenz von Widersprüchen und Konflikten in einer komplexen Gesellschaft anerkennt und dafür Sorge trägt, daß sie in einer demokratischen Weise gelöst werden, wobei aber die Hegemonie des „Volkes“ und die nationale Unabhängigkeit garantiert bleiben. 


\section{Hin zu neuen sozialen Organisationsformen}

Die Stärkung des „Volkes“ muß von der Autonomie seiner Organisationen ausgehen. Das Modell der Organisationen als Transmissionsriemen für die revolutionären Ziele galt unter historischen Bedingungen, die heute nicht mehr existieren. Unter den neuen Umständen müssen sich die gesellschaftlichen Organisationen auf widersprüchliche Bereiche einlassen, eventuell auch konträr zu der vom Staat diktierten Politik. In einem ersten Schritt müßten die schon existierenden Organisationen zu einer solchen Autonomie angeregt werden. Zum Beispiel sollte die für die Frauen bereits sehr wichtige Föderation Kubanischer Frauen in feministischer Perspektive die fortdauernde geschlechtsspezifische Unterdrückung angreifen, die mit der neuen wirtschaftlichen Entwicklung, die die weibliche Arbeitskraft abwertet, noch zunehmen dürfte.

Ähnliches gilt für die Gewerkschaften. In einer subventionierten Wirtschaft und durch ein paternalistisches Arbeitsrecht geschützt, genügte es für die Gewerkschaften zurückhaltend zu agieren. In dem Maße, wie das neue Akkumulationsmodell die Ausbeutung der Arbeitskraft intensiviert und ihre Reproduktionskosten erhöht, müssen die Beschäftigten durch eine kämpferische Gewerkschaftsbewegung vertreten werden, die sich aller Druckmittel, einschließlich des Streiks bedienen kann.

Dies hat seinen Preis. So könnte z.B. eine kämpferische Gewerkschaft einen ausländischen Kapitalanleger, der an kurzfristiger Gewinnmaximierung interessiert ist, abschrecken. ${ }^{6}$ Aber das ist ein unumgänglichen Preis, und außerdem ist diese Art von Kapital nicht unbedingt dasjenige, welches für ein Land interessant sein sollte, das noch andere wirtschaftliche Vorteile hat als nur billige und genügsame Arbeitskräfte.

Als zweiter Schritt bleibt festzuhalten, daß das Potential gesellschaftlicher Organisation im heutigen Kuba nicht durch die traditionellen Massenorganisationen erschöpft ist. Seit fünf Jahren kann in der kubanischen Gesellschaft das Entstehen von unterschiedlichen Vereinigungen beobachtet werden, einige von ihnen mit einer starken öffentlichen Wirkung und dem Ziel eines erneuerten Sozialismus und der Erhaltung der nationalen Unabhängigkeit: Bürgervereinigungen, Nicht-Regierungs-Organisationen, akademische Institute, Gemeindebewegungen etc. Sie behandeln Themen wie neue Visionen von Entwicklung, Umweltschutz, Geschlechterverhältnisse, Bür-

6 Anstelle einer Illustration folgende Anekdote: als das erste Hotel der Kette Meliá in Kuba eröffnet wurde, weigerte sich die spanische Leitung zunächst, eine gewerkschaftliche Vertretung anzuerkennen. Nach Auskunft eines Kubaners aus dem mittleren Management akzeptierten die Spanier die Gewerkschaft schließlich mit der Begründung, daß eine gut organisierte Gewerkschaft viele Aktivitäten vereinfachen könne (Granma 10.April 1991). Dieser Konsens ist beunruhigend. Allerdings haben die Gewerkschaften bei den Reformen den größten Mut gezeigt. Ich habe ihre Rolle zum Teil in Dilla (1996a) beschrieben. 
gerbeteiligung, lokale Kulturen etc. Vor allem die Gemeindebewegungen haben versucht, praktische Alternativen zur Entwicklung und zum Zusammenleben aus der Gemeindeperspektive heraus aufzuzeigen, und damit die traditionellen Gegensätze zwischen Markt und Staat, die die öffentliche Diskussion in Kuba über Jahre hinweg dominierte, zu überwinden (vgl. Dilla/Fernandez/Castro 1997).

Paradoxerweise wurden allen diesen Bewegungen bürokratische Begrenzungen auferlegt. Die Parteiführung veröffentlichte ein Dokument, in dem sie erklärte, daß man beabsichtige, diese Vereinigungen in eine ,Sozialistische Zivilgesellschaft" einzubinden, wobei aber nie definiert wurde, was denn die Kritierien für den Einschluß in diese sozialistische Zivilgesellschaft sein sollten, so daß die neuen Vereinigungen der Willkür der Bürokratie ausgeliefert blieben (vgl. Granma 27. März 1996). Gerechtfertigt wurde diese Politik sowie auch andere restriktive Maßnahmen gegenüber solchen Vereinigungen, mit der US-amerikanischen Interventionspolitik, die die Zivilgesellschaft ähnlich wie in Osteuropa als ein Mittel zur Aufweichung des kubanischen Systems betrachtet. Eine solche Interventionsstrategie existiert zumindest seit 1980 und sie ist auch Bestandteil des Torricelli-Act (1992) und des Helms-Burton-Act (1996). ${ }^{7}$ Es ist daher verständlich, daß der kubanische Staat versucht, die nationale Souveränität gegen die subversiven Absichten der USA zu schützen und daher entsprechende bürokratische „Filter“ errichtet. Aber es ist paradox, daß Organisationen, die ihre Ablehnung gegenüber der nordamerikanischen Einmischung genauso zum Ausdruck gebracht haben wie der eigene Staat, ebenfalls Grenzen und bürokratische Kontrollen auferlegt werden. Möglicherweise drückt die Reaktion des Staates gegen die „Zivilgesellschaft" nicht nur die patriotische Pflicht der politischen Klasse oder die Weigerung der Bürokratie Kontrollrechte abzugeben aus, sondern auch den Vorsatz, dem internationalen Kapital „ein geordnetes Land“ anzubieten, was mit der Existenz von autonomen und kämpferischen Organisationen unvereinbar wäre. Als letztes muß darauf hingewiesen werden, daß die Stärkung des „Volkes“ sich nicht auf die Wiederbelebung der existierenden Organisationen beschränken kann, sondern auch die Gründung von Vereinigungen der im Akkumulationsprozeß neu entstehenden Akteure einschließen muß. Ein erster paradigmatischer Fall sind hier die vielen Selbständigen. Die Mittellage dieses Sektors bedingt dessen politische Ambivalenz gegenüber der Kontinuität des Sozialismus. Die Mehrheit dieser Selbständigen hängt hauptsächlich von ihrer eigenen Arbeitskraft ab. Auch wenn ihr Einkom-

7 Und es blieb nicht nur bei Vorsätzen. Seit Anfang der 90er Jahre versuchten rechte nordamerikanische Stiftungen nicht nur in die Zivilgesellschaft einzudringen, sondern auch in andere Sektoren wie die der zivilen Beamten, des Militärs und der Staatssicherheit (vgl. dazu Azcuy 1995). 
men in der gegenwärtigen - außergewöhnlichen - Wirtschaftslage relativ hoch ist, sind sie nicht fähig, zu akkumulieren. Ihre Lebensqualität hängt im hohen Maße von den sozialen Dienstleistungen und von den staatlichen Bemühungen um gesellschaftlichen Wohlstand ab. Aber gleichzeitig unterliegen sie aufgrund ihrer Geschäftserfahrungen einem Individualisierungsprozeß und beschränken ihren Blickwinkel auf die Gewinne ,ihres“ Geschäftes. Gewisse soziale Pflichten, wie etwa das Zahlen von Steuern, sehen sie als ungerechtfertigte Hindernisse auf dem Weg zu größerem Reichtum an. Da der letzte Kongreß der Kommunistischen Partei (1997) die Zulassung von kleinen und mittleren Unternehmen ablehnte, wurden gleichzeitig Anreize zur Bildung von Kooperativen oder anderen Verbindungen von Produzenten verhindert.

Ein ähnliches Beispiel sind die Agrarkooperativen, die hauptsächlich in den Unidades Básicas de Producción Cooperativa (UBPC) organisiert sind. Die UBPC wurde 1993 auf staatliche Initiative gegründet, um über eine Dezentralisierung bessere Ergebnisse im Agrarsektor zu erhalten. Fast 20\% des strategisch wichtigen staatlichen Bodens wurde an die Arbeitskollektive abgegeben, was einer der mutigsten Schritte der letzten Jahre in Richtung Vergesellschaftung war. Bei der Schaffung der UBPC mangelte es aber seit Beginn an einer klaren politischen Linie. Zunächst wurden den Kooperativen starke bürokratische Zwänge auferlegt, sie hatten keinen freien Zugang zum Markt und waren an ähnliche bürokratische Vorgaben gebunden wie die staatlichen Unternehmen. Ende 1994 wurde ihnen der Zugang zum Markt ermöglicht, was ohne Zweifel zu einer partiellen Dynamisierung führte (vgl. zu den UBPC Pérez/Torres 1996). Aber ohne weitere politische Maßnahmen wird diese Entwicklung einen Kurs nehmen, der im Gegensatz zu ihrer möglichen Rolle bei der Entwicklung des sozialistischen Eigentums und ihrer möglichen internen Demokratie steht. Die Vermeidung dieser Effekte wird nicht vom wirtschaftlichen Erfolg allein abhängen - auch wenn dieser unabdingbar ist -, sondern vom generellen politischen Rahmen, in den sich die Kooperativen integrieren müssen.

Die unabhängigen Arbeiter - Selbständige, Mitglieder von Kooperativen oder zukünftige Inhaber von kleinen oder mittleren Betrieben - stellen für die politische Klasse, die an eine direkte Kontrolle von oben nach unten gewöhnt ist, eine Herausforderung dar. Die bisherige Politik bestand darin, diese Arbeiter in die bestehenden Gewerkschaften einzugliedern, was aber sowohl für die Gewerkschaften wie für die Selbständigen und KooperativMitglieder dysfunktional ist. Es scheint plausibler, diesen Gruppen eigene Organisationen zuzugestehen, die fähig sind, ihre spezifischen Interessen innerhalb des Systems zu repräsentieren, selbst wenn dies eine neue Form der Politik implizieren würde. Nur mit dieser Stärkung des „Volkes“ in den verschiedenen gesellschaftlichen Sphären wäre es möglich, eine alternative 
Wirtschaftsweise zu entwickeln, die verschiedene Formen einer „Volksökonomie “" ${ }^{8}$ innerhalb eines dezentralisierten Modells kombinieren könnte sei es nun im Rahmen von staatlichem, privatem oder gemischtem Eigentum. Zugleich müßten aber auch Organisationen von Konsumenten geschaffen werden, die - geschützt durch eine entsprechende Gesetzgebung den unsozialen Effekten des Marktes auf das Konsumniveau der Bevölkerung entgegentreten könnten.

Dieses politische Modell müßte auch dem technokratisch-unternehmerischen Sektor einen zwar notwendigen, aber untergeordneten Platz zubilligen. Die Bedeutung dieses aufstrebenden Sektors ergibt sich nicht aus seiner Quantität. In Zahlen ausgedrückt, sprechen wir über nicht mehr als über einige tausend Personen, deren Position in der sozialen Hierarchie noch längst nicht stabil ist, denen es an eigenen Strukturen mangelt und die noch kein Bewußtsein als eigene Schicht entwickelt haben. Die Bedeutung dieses Sektors ist an qualitative Parameter gebunden, vor allem an seine Rolle in den dynamischsten Bereichen der Wirtschaft, was ihm eine gewisse Machtposition gegenüber der politische Klasse und der traditionellen Bürokratie einräumt. Die aufstrebenden Sektoren versorgen die traditionelle Elite mit den wirtschaftlichen Überschüssen, die diese für die Reproduktion ihres politischen Projektes benötigt, umgekehrt garantiert die politische Elite den sozialen Frieden, der für das neue Akkumulationsmodell unabdingbar ist. Und schließlich darf man nicht vergessen, daß die neuen Technokraten und Unternehmer selbst aus dem Schoß der traditionellen Bürokratie entstammen, so daß sie von vornherein mit einem Netz von persönlichen Beziehungen und Ressourcen ausgestattet sind.

Die qualitative Relevanz dieses Sektors zeigt sich auch in seiner Fähigkeit zur ideologischen und kulturellen Reproduktion: dazu ist es lediglich notwendig, sich selbst der Gesellschaft gegenüber als Symbol des persönlichen Erfolgs zu präsentieren. Dies hat Auswirkungen auf die Einstellungen und Verhaltensweisen von breiteren Teilen der Gesellschaft: Verhalten, das früher als Abweichung von der Norm galt (wie z.B. Korruption), wird jetzt als Zeichen des Erfolges oder wenigstens als legitimes Mittel des Überlebens betrachtet.

Hier, und nicht in den unbedeutenden Dissidentengruppen, mit denen das System ohne große Probleme leben kann, befindet sich die soziale Basis für einen kubanischen Thermidor oder was in Zukunft eine noch größer Gefahr darstellen könnte: die Wurzel einer tropischen Mafia, die schon angefangen hat, Einfluß zu gewinnen.

8 Rein funktional verstehe ich hier unter „Volksökonomie“ wirtschaftliche Aktivitäten, die von Einzelnen oder von Kollektiven ausgeführt werden, die bei ihrer Reproduktion primär von ihrer eigenen Arbeitskraft abhängen und die ihre Arbeit auf der Grundlage solidarischer Prinzipien organisieren (vgl. auch Corraggio 1994). 
Die wichtigste Grenze für die Entwicklung des technokratisch-unternehmerischen Sektors zu einem neuen hegemonialen Block ergibt sich durch den Fortbestand einer Allianz der breiteren sozialen Schichten mit der politischen Führung, die aus der Revolution hervorging. Das schon erwähnte vorsichtige Vorgehen bei den Reformen und der Marktöffnung - unabhängig von seiner technischen Bewertung - zeugt von der Permanenz dieses grundsätzlichen sozialen Kompromisses. Gerade angesichts der USamerikanischen Feindseligkeiten, die die Wahrung der nationalen Einheit verlangen, ist dieser Kompromiß nicht verhandelbar.

Insgesamt ist der aktuelle Stand der ökonomischen Reformen in Kuba noch durch eine starke Teilung der wirtschaftlichen Aktivitäten und durch fragmentierte Märkte charakterisiert, was die horizontalen Beziehungen zwischen den verschiedenen Komponenten selbst innerhalb eines einzelnen Sektors erschwert. Allerdings werden diese strukturellen Bedingungen der kubanischen Wirtschaft durch die Kraft des Marktes bald beseitigt werden, wenn es nicht zu einer Erneuerung der gesellschaftlichen Blöcke kommt; deren autonome Organisierung setzt aber institutionelle und normative Veränderungen voraus, die allerdings nichts mit der von den USA geforderten „Liberalisierung“ des politischen Systems zu tun haben.

\section{Institutionalisierung und demokratischer Aufbau}

Das erste Glied in dieser Kette müßte eine effizientere und stärker partizipatorische Verwaltung der Gemeinden sein. Hier wäre der erste Ort für eine Abstimmung von Interessen und Verhandlungen. Die lokalen Räume in Kuba sind längst nicht mehr Ausdruck einer zentral geplanten, ausgeglichenen Regionalentwicklung. Statt dessen erfahren sie durch ihre unterschiedliche Beziehung zu der vom Weltmarkt vorgegebenen wirtschaftlichen Dynamik eine höchst ungleiche Entwicklung. Touristische Zentren, Bergbaugebiete und Freihandelszonen sind die neuen Variablen, die den nationalen Raum fragmentieren und das Lokale diversifizieren (vgl. Dilla 1996b). Die lokalen Gesellschaften transformieren sich durch die Entstehung von neuen ökonomischen und sozialen Akteuren. In diesem Kontext bleibt wenig Raum für die traditionelle Art der Entscheidungsfindung entsprechend den Vorgaben der zentralen Wirtschaftslenkung. Ein zukünftiger Planungsprozeß müßte dezentral und pluralistisch sein und an diesen lokalen Einheiten ansetzen.

Aber auch auf der Makroebene ist eine Veränderung in der Konstitution der Repräsentativorgane notwendig. Bisher wurde bei den Wahlen kein Wettbewerb von Kandidaten akzeptiert. Dies hat auf der einen Seite dazu geführt, daß bei den Bezirksregierungen die ansässigen Arbeiter aber auch die Frauen nur mangelhaft repräsentiert sind, auf der anderen Seite erodierten 
die Repräsentativorgane zu Fiktionen. Eine Reform dieser Institutionen sollte gleichzeitig die Anforderungen der territorialen, sektoralen und interessensgeleiteten Repräsentation erfüllen. Dabei müßte natürlich auch darauf geachtet werden, daß die gesetzlichen Vorgaben, die den repräsentativen Organen in jedem Bezirk die größte staatliche Macht verleihen, eine reale Gültigkeit erhalten. Bis heute sind die repräsentativen Organe an der Gesetzgebung nur schwach beteiligt - mit wenigen Sitzungstagen im Jahr und einem beunruhigenden Einheitsvotum bei einem Großteil der diskutierten Themen.

Nicht minder relevant sind Freiheiten, Rechte und Pflichten der Bürger, die vom Gesetz geschützt und institutionell unterstützt werden müssen. Die kubanische Revolution hat zwar eine Vielzahl von sozialen und ökonomischen Rechten aufgestellt, die sich nicht in der „Magie des Marktes“ auflösen dürfen und die als genuine revolutionäre Errungenschaften verteidigt werden müssen. Gleichzeitig gab es aber nur einige magere und ungenaue Deklarationen zu politischen und bürgerlichen Rechten. Dies hat zu bedauerlicher Willkür geführt, worunter die Individual- und Kollektivrechte, die öffentliche Debatte und die Ideenvielfalt gelitten haben. Hier sollte man daran erinnern, wie der klassische Marxismus im Kommunistischen Manifest die Gesellschaft beschrieb, die an die Stelle des Kapitalismus treten sollte: ,... eine Vereinigung, in der die freie Entfaltung eines jeden die Bedingung für die freie Entfaltung aller ist."

Die Kommunistische Partei Kubas sollte sich einem derartigen Wandel nicht entgegenstellen: sie sollte ihn vielmehr vorantreiben. Nur sie, als die zentrale Organisation des politischen Systems, mit mehr als einer halben Million politisch geschulter und dem Sozialismus zugewandter Mitglieder, könnte die unumgänglichen Veränderungen mit den geringsten Gefahren in Gang bringen und in diesem Proze $ß$ die ursprüngliche Macht des Volkes konsolidieren. Aber dies impliziert die Transformation der Partei selbst. Eine neue Form der Organisation und Funktion müßte gefunden werden, die eher den verschiedenen Interessen entspricht, die in den sozialen und politischen Bereichen existieren und anerkannt werden müssen. In einem optimalen Szenario würde dies zu einer demokratischeren Partei führen, die auf der Basis einer grundsätzlichen Einheit über die strategischen Positionen offen gegenüber Diskussionen und unterschiedlichen Tendenzen ist. Dies könnte auch zu einem Mehrparteiensystem führen, insbesondere wenn die Kommunistische Partei ihre Rolle als Avantgarde aufgeben würde. Die Verschiebung des politischen Systems in die skizzierte Richtung könnte dabei die Entstehung von Parteien begünstigen, die sich dem System gegenüber loyal verhalten.

Das kubanische Volk hat in 40 Jahren einen hohen Preis für die Sünde bezahlt, ein alternatives Projekt mit sozialistischer Ausrichtung und nationa- 
ler Unabhängigkeit dort aufzubauen, wo die USA immer ihren Hinterhof gesehen haben. Viele Jahre lang mußte es diesen Preis für die nicht zu leugnenden Vorteile der sowjetischen Unterstützung zahlen. Heute zahlt das Volk einen doppelten Preis für das Beharren auf seinen Zielen, indem es mit einer einzigartiger Härte erneut die historische Tragödie vom Sozialismus in einem Land erfährt. Doch ist das Überleben eines erneuerten Sozialismus, der in einer globalen antikapitalistischen Strategie verwurzelt ist, nicht unmöglich. Allerdings wird dieses Überleben nicht von den einst beschworenen ,allgemeinen Gesetzen der Geschichte“ garantiert, auch eine Restauration des Kapialismus ist möglich. In diesem Fall müßte der in den letzten 40 Jahren bezahlte Preis als eine Investition in eine künftige linke Alternative betrachtet werden. Und deshalb lohnt es sich weiter voranzugehen, Don Quijotes Ermahnung an seinen Schildknappen folgend - trotz des Protestes von Ex-Bürokraten, die sich in Unternehmer verwandelt haben, von Ex-Dogmatikern, die sich in Liberale verwandelten oder einfach von denjenigen, die glauben, daß wir jetzt doch bei dem schon so häufig prognostizierten Ende der Geschichte angekommen seien.

Aus dem Spanischen übersetzt von Hans-Jürgen Burchardt

\section{Literatur}

Anuario de 1989 del Comité Estatal de Estadisticas, Havanna.

Azcuy, Hugo (1996): La reforma de la constitución socialista de 1976, in: H. Dilla (Hrsg.), La democracia en Cuba y el diferendo con los Estados Unidos. CEA, Havanna.

Azcuy, Hugo (1995): Estado y Sociedad Civil en Cuba, in: Temas Nr. 4, Oktober/November Havanna.

Beruff, Alejandro (1997): Las finanzas internas de Cuba, in: CEEC Balance de la economía cubana. Havanna.

Comunicado acerca de los resultados del Censo de Poblaciòn y viviendas 1981, Havanna, August 1983.

Corraggio, José Luis (1994): De la economía informal a la economía popular, Nueva Sociedad Nr. 131, Mai-Juni.

Dilla, Haroldo (1996): Cuba: ¿Cuál es la democracia deseable? in: ders. (Hrsg.) La democracia en Cuba y el diferendo con los Estados Unidos, CEA, Havanna.

Dilla, Haroldo (1996a): Comunidad, participación y socialismo: reinterpretando el socialismo cubano, in: ders. (Hrsg.), La Participación en Cuba y los retos del futuro, CEA, Havanna.

- (1996b) Municipios y construcción democrática en Cuba, Perfiles Latinoamerikanos, Flacso-Mexiko.

Dilla, H; Fernandez, A.; Castro, M.(1997): Movimientos barriales en Cuba, San Salvador.

García, Tania (1996) ¿Cuentapropismo o economía popular? Vortrag im Seminar „Municipios, Economía Local y Economía Popular“. CEA, 7-8 März 1996.

Giddens, Anthony (1997): Jenseits von Links und Rechts, Frankfurt/M.

Held, David (1992): Modelos de democracia. Alianza Editora, Mexico.

Llamamiento al IV Congreso del Partido, in: Cuadernos de Nuestra América, Juni-Dezember 1990.

Pérez, Niurka; Torres, Cary (1996): UBPC: hacia un nuevo proyecto de participación, in H.Dilla (Hrsg.) La Participación en Cuba y los retos del futuro, CEA, Havanna.

Togoros, Vivian (1997): Enfoque social del desempeño de la economía cubana en 1996, in: CEEC Balance de la economía cubana, Havanna.

Valdés, Juan (1997): Procesos agrarios en Cuba. Editorial de Ciencias Sociales, Havanna. 